\title{
Pathologic Stage I Gastroesophageal Junction Adenocarcinoma AJCC v8
}

National Cancer Institute

\section{Source}

National Cancer Institute. Pathologic Stage I Gastroesophageal/unction

Adenocarcinoma A/CC v8. NCI Thesaurus. Code C133565.

Stage I includes: IA: (T1a, N0, M0, G1); (T1a, N0, M0, GX); IB: (T1a, N0, M0, G2); (T1b, N0, M0, G1-2); (T1b, N0, M0, GX); IC: (T1, N0, M0, G3); (T2, N0, M0, G1-2). T1a: Tumor invades the lamina propria or muscularis mucosae. T1b: Tumor invades the submucosa. T1: Tumor invades the lamina propria, muscularis mucosae, or submucosa. T2: Tumor invades the muscularis propria. N0: No regional lymph node metastasis. M0: No distant metastasis. G1: Well-differentiated. GX: Grade cannot be assessed. G2: Moderately differentiated. G3: Poorly differentiated, undifferentiated. (AJCC 8th ed.) 